\title{
IN VIVO OPTICAL MOLECULAR IMAGING: PRINCIPLES AND SIGNAL PROCESSING ISSUES
}

\author{
Jong Chul Ye ${ }^{1}$,Kevin Webb ${ }^{2}$, Rick Millane ${ }^{3}$, and Charles Bouman ${ }^{2}$ \\ ${ }^{1}$ Dept. of BioSystems, Korea Advanced Institute of Science and Technology, Daejon, Korea \\ ${ }^{2}$ Purdue University, School of Electrical and Computer Engineering, West Lafayette, IN, USA \\ ${ }^{3}$ Dept. of Electrical \& Computer Engineering, Univ. of Canterbury, Christchurch, New Zealand
}

\begin{abstract}
In vivo optical molecular imaging involves the use of light emitting tracers combined with sophisticated sensing modalities to perform in vivo imaging of genetic and molecular information. In contrast to the classical diagnostic imaging tools which image the end effects of the diseases, optical molecular imaging could enhance our knowledge of biological phenomena, monitor genetic expression and the alteration of cells, and lead to earlier detection of diseases. With the development of exotic molecular probes with easily detectable bioluminescence and fluorescence labels, optical molecular imaging has emerged as an important new field within biomedical imaging. This paper reviews this stateof-the-art imaging technology and signal processing issues to monitor molecular and cellular events in living organism.
\end{abstract}

\section{INTRODUCTION}

The goal of molecular imaging is monitoring biological processes at the cellular and molecular level in vivo. Unlike conventional anatomical imaging modalities, molecular imaging visualizes the cellular and molecular pathways and in vivo mechanisms of disease. Such molecular changes occur in disease much earlier before a mass becomes visible, hence molecular imaging can be used for early treatment of cancers, significantly reducing the mortality rate. Due to the promising outlook of molecular imaging, major diagnostic imaging companies such as GE, Siemens and Philips have made commitments to molecular imaging even at this early stage in its development.

Molecular imaging is a multidisciplinary field. The recent emergence of molecular imaging is largely due to the unprecedent advances in molecular biology, the availability of exotic probes, and the successful development of new types of small animal imaging devices and sophisticated reconstruction algorithms. In molecular imaging, once a specific imaging target is defined, a ligand with high affinity to the target should be found. This can be antibodies, or recombination proteins. Depending on the imaging modality, a contrast agent or label should be found that binds to the ligand.

Currently, the term "molecular imaging" has been used to refer to various scales of in vivo bio-medical imaging. For the biologist, molecular imaging may be synonymous to in vivo cell imaging using fluorescence microscopy, where exotic fluorescence probes are used to monitor the cell cycle, signal pathways, gene expressions, nuclear trafficking, etc. One of the main applications of cell level molecular imaging is high content screening (HCS) to accelerate the drug discovery process. For the radiologist, the term "molecular imaging" implies whole body imaging using existing imaging modalities such as positron emission tomography (PET), magnetic resonance imaging (MR), optical imaging, etc. This group is primarily interested in reporter gene imaging which can be used for cell marking, gene therapy imaging, transgenic animal imaging, molecular interactions, etc.

Specialized optical imaging devices for small animal imaging have been developed recently. These have used either bioluminescence or fluorescence imaging techniques to monitor whole body, providing low cost and the versatility of optical spectroscopy.

In this paper, we discuss the common link between cell level and whole body level optical molecular imaging. Our focus is more on the imaging system and signal processing issues for optical molecular imaging than molecular probe design problems that have been extensively reviewed elsewhere [1].

\section{GENERATION OF LIGHT}

Bio-luminescence is the ability of living organ to emit light. Depending on its origin, it is called either chemiluminescence (or bioluminescence in a narrow sense) or fluorescence. An example of chemiluminescence is generated by the firefly luciferase, which produces light through oxidation of luciferin. In mammalian species, the luciferase gene does not exist, so it should be transfected by genetic engineering. The luciferase gene then works as a marker gene for gene expression. Since there is no background signal, it 
can be used to detect much lower levels of light with a relatively simple detection system [2]. This simplicity and high sensitivity of a bioluminescence system has been the major contributor in the success of the start-up company Xenogen. However, since luciferase requires genetic transfection, it is not possible to use for an application in human studies.

Fluorescence is a process in which molecules absorb light and emit light of a longer wavelength after a brief interval. In principle, fluorescence is the result of a three-stage process. In the first stage, an excitation photon of energy $h \nu_{E X}$ is absorbed by a fluorophore, where $h$ is Planck's constant and $\nu$ is frequency, elevating charge from the ground to the excited state. The fluorophore then relaxes to the lowest vibrational energy level of the first excited state, and then the excited fluorophore returns to the ground state with a characteristic time constant, resulting in emission of a photon with longer wavelength and energy $h \nu_{E M}$.

Several parameters are important in describing the fluorescence phenomena, such as Stoke shift and fluorescence life time. The Stoke shift is defined as the energy or frequency difference between the excitation and emission photons, and a larger Stoke shift gives a greater detection accuracy. The fluorescence lifetime $\tau$ denotes the characteristic time that a molecule remains in an excited state before returning to the ground state. The lifetime is an important characteristic of the fluorophore and can be used to distinguish between fluorophores with similar spectral characteristics or signals from the background auto-fluorescence.

\section{OPTICAL MOLECULAR PROBES}

Fluorescence imaging agents have been extensively studied for biological applications. For cell biology, fluorescent indicators currently exist for calcium, $\mathrm{pH}$, ATP, membrane potential, and several neurotransmitters [3]. Recently a new type of naturally fluorescent protein such as green fluorescence protein (GFP), obtained from jellyfish Aequorea victoria, and its two spectral variations, have been widely used for in vivo imaging of cells [3]. Indocyanine green (ICG) is another useful fluorophore with near-infrared (NIR) range emission. The relatively low absorption and scatter of living tissue in the near-infrared (NIR) region of the electromagnetic spectrum makes near-infrared fluorescence probes such as ICG important for in vivo imaging. Furthermore, such an imaging agent can be coupled to antibodies or proteins and targeted specifically to tumor cells. Another improvement of imaging probes is the advance of "smart probes" that can only be detected once they have interacted with their substrate [1].

Recently, inorganic nano-particles or fluorescent semiconductor nanocrystals (quantum dots), have attracted much attention from the biomedical imaging community as a potentially powerful contrast agent [4]. Important properties of quantum dots (QDs) include broadband absorption spectra and a relatively narrow emission band, which is ideal for imaging applications.

\section{MOLECULAR CELL IMAGING}

\subsection{Detection System}

The popular detection mechanism for cell imaging is laser scanning confocal microscopy (LSCM). When fluorescent specimens are imaged using a conventional wide-field optical microscope, out-of-focus plane fluorescence emitted by the specimen often interferes with the features in the region of interests, which reduces the spatial resolution of the microscope images. In a confocal microscope, the unwanted out-of-focal plane light is removed with the aperture near the detector. Unlike a conventional widefield microscope in which the entire specimen is illuminated by the light source, in confocal microcopy the illumination is focused only in a small section of the specimen. This increases the scan time for a specimen, relative to a wide field microscope. In order to achieve a fast scan, disk scanning confocal microscope (or Nipkow disk system) employs a spinning disk with multiple small holes between the light source and specimen.

Multiphoton (usually two photon) confocal microscopy is based on nonlinear two photon excitation [5], which occurs by the simultaneous absorption of two photons each having half the energy required for fluorescence excitation. Since efficient two-photon excitation requires a high spatial and temporal concentration of photons, it results in a confocal effect at the focal spot without using any means such as a pinhole. Furthermore, low energy NIR excitation can be used to penetrate deeply within the specimen.

\subsection{Signal Processing Issues}

With ever increasing computational power, algorithmic approaches have been investigated to replace confocal microscopy. In so called "computational sectioning", multiple cell images are taken using different foci [6] or views [7]. Then, using deconvolution techniques or computed tomography algorithms, three dimensional images can be obtained. Another promising approach is using a structured light illumination pattern to minimize the out-of-focus interferences [8].

Another important signal processing issue is signal separation. For example, biologists often introduce two or more probes to simultaneously monitor different biochemical processes, resulting in overlapped emission spectra. Furthermore, many important fluorescence proteins have excitation and emission curves that closely overlap each other, hence the classic bandpass filter approaches does not work effectively. In fact, a similar multi-spectral imaging problem has been widely studied for geological remote sensing applications, in which the problem can be basically reduced to a classifier design problem. If the reference spectra are not known, sophisticated classification algorithms such as principal component analysis (PCA), independent component analysis (ICA) and k-mean clustring can be used for spectral 
separation [9]. However, if reference spectra are available, much simpler linear algorithm can be used. For example, for fluorescence proteins such as CFP, GFP, and YFP , the measured spectrum $S(x, y ; \lambda)$ at the spatial location $(x, y)$ can be represented by

$$
\begin{aligned}
S(x, y ; \lambda)= & w_{1}(x, y) C F P(\lambda)+w_{2}(x, y) G F P(\lambda) \\
& +w_{3}(x, y) Y F P(\lambda)
\end{aligned}
$$

where $C F P(\lambda), G F P(\lambda)$ and $Y F P(\lambda)$ denote the spectra of CFP, GFP, and YFP, respectively; and $w_{i}(x, y), i=$ $1, \cdots, 3$ denotes the corresponding weights. The signal separation problem is then estimating $w_{i}(x, y), i=1, \cdots, 3$ using (1).

\section{IN VIVO SMALL ANIMAL IMAGING}

\subsection{Diffusion Approximation}

Optical diffusion tomography (ODT) is a sensitive and relatively low cost imaging modality that has been studied quite extensively to the preclinical stage[2, 10]. A major difficulty with optical imaging is that the signal within tissue experiences significant scatter. However, use of a forward model that describes scatter and absorption can form the basis of an image. In highly scattering media such as tissue, the photon attenuation does not follow Beers-Lambert law (exponential decay with distance). With sufficient scatter, the photons can be treated as particles which elastically scatter through the random medium. The theoretical framework for this model is Boltzmann transport theory. If scatter dominates absorption, which is true for soft tissue in the 650-1300 nm range, the Boltzmann transport equation can be approximated using the diffusion equation. More specifically, the photon flux $\psi(\mathbf{r}, t)$ at $\mathbf{r} \in \mathbb{R}^{3}$ satisfies

$$
\frac{1}{c} \frac{\partial}{\partial t} \psi(\mathbf{r}, t)-\nabla \cdot(D(\mathbf{r}) \nabla \psi(\mathbf{r}, t))+\mu_{a}(\mathbf{r}) \psi(\mathbf{r}, t)=-s(\mathbf{r}, t),
$$

where $s(\mathbf{r}, \mathbf{t})$ is the time varying photon source density, and the diffusion constant is given by $D(\mathbf{r})=1 / 3\left(\mu_{a}(\mathbf{r})+\mu_{s}^{\prime}(\mathbf{r})\right)$, with $\mu_{a}(\mathbf{r})$ the absorption coefficient and $\mu_{s}^{\prime}(\mathbf{r})$ the reduced scattering coefficient. In bioluminescence imaging using luciferase, the imaging objective is to find the source distribution $\mathbf{s}(\mathbf{r}, t)$ from the flux measurement $\psi(\mathbf{r}, t)$ at the detector locations. With fluorescence, the situation is more complicated since the excitation and emission spectra are not identical and the optical parameters $\mu_{a}$ and $\mu_{s}^{\prime}$ are wavelength dependent. Hence, the emission photon flux envelope $\psi_{m}(\mathbf{r}, t)$ from the excited fluorophore should be calculated again by another diffusion equation using (2) with the different optical parameters $\mu_{a_{m}}$ and $\mu_{s_{m}}^{\prime}$ [11]. Here, the equivalent source term $\mathbf{s}(\mathbf{r}, t)$ should come from the fluorophore distribution, given by

$$
\mathbf{s}(\mathbf{r}, t)=\psi(\mathbf{r}, t) \eta \mu_{a_{f}}(\mathbf{r}) e^{-t / \tau(\mathbf{r})}
$$

where $\psi(\mathbf{r}, t)$ is the excitation photon flux, $\eta \mu_{a_{f}}$ is the effective quantum yield after absorption, and $\tau$ denotes the fluorescence lifetime. For fluorescence molecular imaging, the imaging objective is to find the distribution of $\mathbf{s}(\mathbf{r}, t)$ and its intensity. For lifetime imaging, we are also interested in reconstruction of $\tau(\mathbf{r})$.

\subsection{Signal Processing Issues}

Currently, there are several ODT implementations, continuous wave (CW), time domain (TD) [12] and frequency domain approaches [10]. Each has merits, as we discuss.

In continuous wave approaches, unmodulated light is illuminated over the phantom, resulting in time-invariant photon distribution (hence, $\partial / \partial t \psi(\mathbf{r}, t)=0$ in (2)). Since bioluminescence emission is relatively slowly varying with time after the injection of luciferin, it can be modeled using the $\mathrm{CW}$ approach. The problem can be reduced to a simple linear inverse problem under some assumptions. More specifically, if all the optical parameters $\mu_{a}(\mathbf{r})$ and $D(\mathbf{r})$ are known, the spatially varying photon flux at detector location $\mathbf{r}$ is given by

$$
\phi(\mathbf{r})=\int g\left(\mathbf{r}, \mathbf{r}^{\prime}\right) s\left(\mathbf{r}^{\prime}\right) d \mathbf{r}
$$

where $g\left(\mathbf{r}, \mathbf{r}^{\prime}\right)$ denotes the known Green's function for (2), and $s\left(\mathbf{r}^{\prime}\right)$ is the unknown source magnitude at $\mathbf{r}^{\prime}$ (whether it comes from bioluminescence or fluorescence). Note that in (4), the Green's function corresponds to a blurring kernel or point spread function (PSF). Therefore, the problem of estimating the source $\mathbf{s}(\mathbf{r}, t)$ becomes a relatively simple deconvolution problem. Xenogen's successful IVIS system is built on an assumption that the Green's function $g\left(\mathbf{r}, \mathbf{r}^{\prime}\right)=\delta\left(\mathbf{r}-\mathbf{r}^{\prime}\right)$. Recently, more sophisticated deconvolution approaches have been proposed, by explicitly calculating the Green's function using (2) with the help of an computed tomography [13], or blindly estimate the source distribution without knowing the PSF [14]. Note that in CW approaches the fluorescence lifetime information $\tau(\mathbf{r})$ in (3) disappears because of time integration.

The time-domain approach uses an short laser pulse, and the emitted fluorescence signals are detected using, for example, a PMT to obtain the temporal point spread function (TPSF). Using the time-domain diffusion equation (2) with (3), we can reconstruct the absorption and scattering parameters for the tissue, as well as the fluorophore distribution and lifetime parameters. One commercial implementation of the time-domain approach is by Advanced Research Technologies Inc., who signed contract with GE Healthcare for the exclusive distribution of systems.

In the frequency domain approach, with a single modulation frequency, a complex flux is measured. Usually, a NIR laser is modulated at angular frequency $\omega$, and a photodiode or PMT can be used with heterodyne detection technique to obtain the phase and intensity from the photon flux 
modulation envelope. CCD implementation of the detector can also be accomplished by modulating an image intensifier, providing homodyne or heterodyne detection of the modulated photon flux. Then, the fluorescence parameters can be obtained using frequency domain version of (2) and (3).

The most rigorous inversion approach for time or frequency domain approaches use iterative reconstruction algorithms. For example, maximum a posteriori (MAP) estimation in a Bayesian framework has been very successful for frequency domain imaging, where shot noise detection statistics and a priori information were incorporated $[15,11]$. In order to accelerate the computation time, more sophisticated and powerful algorithms such as multigrid approaches have been used $[15,16]$.

\section{CONCLUSIONS}

Molecular imaging allows monitoring of gene expression and the alteration of cells, providing the potential for earlier detection of disease. The two principal components are the molecular probe and the imaging system. Molecular probes are designed by incorporating detectable labels into a ligand with high affinity to the target. Imaging modalities are then designed to detect the label with high sensitivity and resolution. Molecular imaging activities encompasses both in vivo cell imaging and whole body imaging. Optical imaging has attracted extensive research attention because it not only provides a link between the two levels of imaging, but also permits spectroscopic information in a safe and inexpensive instrument. With the advance of new types of optical probes and breakthroughs in inversion algorithms, in vivo optical molecular imaging has great potential to become a key imaging tool for the early detection of cancer.

\section{REFERENCES}

[1] Ralph Weissleder, "Scaling down imaging: Molecular mapping of cancer in mice," Nature Reviews Cancer, vol. 2, no. 1, pp. 1-8, January 2002.

[2] C.H. Contag, S.D. Spilman, P.R. Contag, M. Oshiro, B. Dennery, and et al, "Visualizing gene expression in living mammals using a bioluminescent reporter," Photochem Photobiol, , no. 66, pp. 523-531, 1997.

[3] "Molecualr probes handbook," in http://www.probes.com/handbook/.

[4] M. Bruchez, M. Moronne, P. Gin, S. Weiss, and A.P. Alivisatos, "Semiconductor nanocrystals as fluorescent biological labels," Science, , no. 281, pp. 20132016, September 1998.

[5] W. Denk, J.H. Strickler, and W.W. Webb, "2-photon laser scanning fluorescence microscopy," Science, pp. 73-76, 1990.
[6] J.A. Conchello, "Superresolution and convergence properties of the expectation-maximization algorithm for maximum-likelihood deconvolution of incoherent images," J. Opt. Soc. Am. A, , no. 3, pp. 2609-2620, 1998.

[7] S. Kawata, 0. Nakamura, and S. Minami, "Optical microscope tomography. I. support constraint," J. Opt. Soc. Am. A, , no. 1, pp. 292-297, January 1987.

[8] M. Neil, R. Juskaitis, and T. Wilson, "Method of obtaining optical sectioning by using structured light in a conventional microscope," Opt. Letters, , no. 24, pp. 1905-1907, December 1997.

[9] R.O. Duda, P. E. Hart, and David G. Stork, Pattern Classification, John Wiley and Sons, 2nd edition, 2001.

[10] C.L. Hutchinson, T.L Troy, and E.M Sevick-Muraca, "Fluorescence lifetime determination in tissues and other random media from measurement of excitation and emission kinetics," Applied Optics, , no. 35, pp. 2325-2332, 1996.

[11] A. B. Milstein, S. Oh, K. J. Webb, C. A. Bouman, Q. Zhang, D. A. Boas, and R. P. Millane, "Fluorescence optical diffusion tomography," Appl. Opt., vol. 42, no. 16, pp. 3081-3094, June 2003.

[12] R. Barbour, H. Graber, Y. Wang, J. Chang, and R. Aronson, "A perturbation approach for optical diffusion tomography using continuous-wave and timeresolved data," Proc. of SPIE Conf. on Photon Migration and Imaging in Random Media and Tissues, vol. 1888, pp. 87-120, May 1993.

[13] Ge Wang and E. A. Hoffman et al., "Development of the first bioluminescent CT scanner," Radiology, vol. 229, pp. 566, 2003.

[14] Stuart M. Jefferies, Kathy J. Schulze, Charles L. Matson, and Kurt Stoltenberg, "Blind deconvolution in optical diffusion tomography," OPTICS EXPRESS, vol. 10, no. 1, pp. 46-53, January 2003.

[15] J. C. Ye, C. A. Bouman, K. J. Webb, and R. P. Millane, "Nonlinear multigrid algorithms for Bayesian optical diffusion tomography," IEEE Trans. Image Processing, vol. 10, no. 5, pp. 909-922, June 2001.

[16] S. Oh, A. B. Milstein, C. A. Bouman, and K. J. Webb, "A general framework for nonlinear multigrid inversion," IEEE Trans. on Image Processing, , no. 1, pp. 125-140, January 2005. 